Research Article

\title{
Multiconsensus of Nonlinear Multiagent Systems with Intermittent Communication
}

\author{
Jie Chen $\mathbb{D}^{1},{ }^{1}$ Guang-Hui Xu $\mathbb{D}^{2}{ }^{2}$ and Liang Geng ${ }^{1}$ \\ ${ }^{1}$ Hubei University of Technology, School of Science, Wuhan 430068, China \\ ${ }^{2}$ Hubei University of Technology, School of Electrical and Electronic Engineering, Wuhan 430068, China \\ Correspondence should be addressed to Guang-Hui Xu; xuguanghui29@163.com
}

Received 18 May 2020; Accepted 11 August 2020; Published 27 August 2020

Guest Editor: Hou-Sheng Su

Copyright $\odot 2020$ Jie Chen et al. This is an open access article distributed under the Creative Commons Attribution License, which permits unrestricted use, distribution, and reproduction in any medium, provided the original work is properly cited.

Compared with single consensus, the multiconsensus of multiagent systems with nonlinear dynamics can reflect some real-world cases. This paper proposes a novel distributed law based only on intermittent relative information to achieve the multiconsensus. By constructing an appropriate Lyapunov function, sufficient conditions on control parameters are derived to undertake the reliability of closed-loop dynamics. Ultimately, the availability of results is completely validated by these numerical examples.

\section{Introduction}

Multiagent systems have attracted much attention in the field as computer science, vehicle systems, unmanned aerial vehicles, or formation flight of spacecraft since 2009. In most of studies on consensus problem, researchers always adopt the same method that makes all agents finally reach the same value in systems [1-5]. Due to various parameters as sudden changes on environment or cooperative tasks in the reality, the purpose of our research also becomes multiple.

In [6], the consensus problem from asynchronous group of the discrete-time heterogeneous multiagent system under dynamic-change interaction topology is discussed and studied briefly. For different agents, two asynchronous consensus protocols are given in this paper. Based on fixed and switching topology, the multiconsensus of first-order multiagent system is discussed. And we assumed that interactions could reach balance between two subnetworks in $[6,7]$. The relationship between multiconsensus ability and the underlying digraph topology is discussed in [8]. For fixed communication networks, the author proposes a consensus protocol which can be applied to two different second-order multiagent systems under the same assumption [9]. By using pinning control method in [10-12], we can obtain some criteria on multiconsensus of networks without assuming the balance of network topology. For multiconsensus problem from discrete-time multiagent systems with stochastic and fixed topologies, the author performs a professional study in [13]. In [14], the author performs the research on multiconsensus control of switching-directed interaction and fixed topology in common linear multiagent systems, based on pinning control techniques, matrix analysis theory, and Lyapunov stability theory. The cluster consensus of multiagent dynamical systems with impulsive effects and coupling delays was investigated in [15], where interactions among agents were uncertain.

In other words, above conclusion on the consensus of multiagent system with nonlinear dynamics is mostly based on common assumption that information is transmitted continuously between all agents, which means that each single agent shares the information with its neighbors without any communication constraints. However, this is not the fact in reality. For instance, all agents can only get the information from their neighbors during certain disconnected time intervals, as a reason of communication restrictions. In [16-23], intermittent control has attracted more attention. For distributed consensus problem from intermittent control of a time-invariant undirected communication topology in the linear multiagent system, the author designs a type of distributed-observer protocols. In [24], the consensus problem on periodical-intermittent 
control of second-order agent networks, which is based on matrix theory, Lyapunov control method, and algebraic graph theory, is discussed. Based on the above discussion and research, this paper mainly studies on some characteristics of the second-order multiagent system, such as multiconsensus. In the same subnet, nonlinear dynamics of all agents are the same, while all agents in different subnet have different dynamics. Multiconsensus indicates that all agents in every subgroup can be consistent. Between different groups, there is no consistent value. The research performed in this paper can be summarized as three points: firstly, the multiconsensus of the multiagent system with nonlinear dynamics is studied. Secondly, a novel multiconsensus law, which is devised through intermittent and relative state information, is more general than other second-order multiconsensus protocol. And, in such a protocol, all agents always need to communicate with their neighbors.

In Section 2 of this paper, the research model has been designed. In Section 3, we perform the study and discussion on the multiconsensus problem of second-order multiagent systems with nonlinear dynamics. In Section 4, two numerical examples are given to prove the effectiveness on the designed protocol. And the conclusion is summarized in Section 5.

\section{Preliminary}

2.1. Algebraic Graph Theory. In general, the communication topology between agents in a multiagent system is described by a directed graph. Let $G=(V, \varepsilon, A)$ be a system communication topology diagram consisting of $\mathrm{N}$ nodes, the vertex set $V=\left\{v_{1}, v_{2}, L, v_{n}\right\}$ is nonempty finite, the edge set $\varepsilon I ́ \mathrm{~V} \times \mathrm{V}$, and a nonsymmetric $A=\left(a_{i j}\right)_{n \times n}$ is nonnegative weighted adjacency matrix. $A=\left(a_{i j}\right)_{n \times n}$ is defined as $a_{i j} \neq 0$ if $\varepsilon_{i j} \in \varepsilon$ and $a_{i j}=0$ otherwise. There are no self-loops, i.e., $a_{i i}=0$. The set of neighbors of agent $i$ is denoted by $N_{i}=\left\{v_{j} \mid \varepsilon_{i j} \hat{\mathrm{I}} \varepsilon\right\}$. A directed path is a sequence of distinct vertices $1,2, \ldots, r$ such that $\left(v_{i}, v_{j}\right) \in \varepsilon, i=1,2, \ldots, r-1$.

2.2. Problem Description. Consider a multiagent system with $n$ agents, $v=\{1,2, \ldots, N\}$. Suppose the multiagent system composed with $p$ subgroup, $v_{p}$ is a set of $p$ subgroup. Note that the corresponding subtopology graph of each subgroup is $\mathscr{G}_{p}$, and the topology diagram of the whole system is $G$. The corresponding numbering sets for each subgroup are $v_{1}=\left\{1, \ldots, l_{1}\right\}, \quad v_{2}=\left\{l_{1}, \ldots, l_{1}+l_{2}\right\}, \quad v_{p}=\left\{l_{1}+l_{2}+\cdots+\right.$ $\left.l_{p-1}, \ldots, N\right\}$, and $l_{1}+l_{2}+\cdots+l_{p-1}+l_{p}=N . \quad v_{i} \neq \phi, \cup_{i=1}^{p}$ $v_{i}=v$, and $v_{i} \cap v_{j} \neq \phi$ for $i \neq j$. For $i \in v$, let $\bar{i}$ denote the subscript of the subset to which the integer $i$ belongs. It is assumed in what follows that each agent knows which cluster it belongs to.

The dynamics of systems is described as follows:

$$
\ddot{q}_{i}(t)=f_{\bar{i}}\left(q_{i}, \dot{q}_{i}, t\right)+u_{i}(t),
$$

where $q_{i}(t) \in R^{n}, \dot{q}_{i}(t) \in R^{n}$, and $u_{i}(t) \in R^{n}$ are the position, velocity, and control input of agent $i$, respectively. The function $f_{\bar{i}}\left(q_{i}, \dot{q}_{i}, t\right) \in R^{n}$, describing the intrinsic dynamics of agent $i$, is continuously differentiable.
In this work, the leader in each group is described by

$$
\ddot{q}_{\bar{i}}(t)=f_{\bar{i}}\left(q_{\bar{i}}, \dot{q}_{\bar{i}}, t\right) .
$$

Definition 1. The multiconsensus control for second-order multiagent systems is said to be achieved if

$$
\begin{aligned}
& \lim _{t \longrightarrow \infty}\left\|q_{i}(t)-q_{\bar{i}}(t)\right\|=0, \\
& \lim _{t \longrightarrow \infty}\left\|\dot{q}_{i}(t)-\dot{q}_{\bar{i}}(t)\right\|=0 .
\end{aligned}
$$
follows:

Firstly, some basic assumptions and lemmas are given as

Lemma 1. There is a constant $\omega \in R$, and $P, Q, M, N$ are matrices with suitable dimensions. Then, the Kronecker product has the following properties:

$$
\begin{aligned}
(1)(P \otimes Q)^{T} & =P^{T} \otimes Q^{T}, \\
(2)(\omega P) \otimes Q & =P \otimes(\omega Q), \\
(3)(P+Q) \otimes M & =P \otimes M+Q \otimes M, \\
(4)(P \otimes Q)(M \otimes N) & =(P M \otimes Q N) .
\end{aligned}
$$

Lemma 2. The linear matrix inequality

$$
\left[\begin{array}{cc}
A(s) & B(s) \\
B^{T}(s) & C(s)
\end{array}\right]>0,
$$

where $A(s)=A^{T}(s), C(s)=C^{T}(s)$, and $B(s)$ depend affinely on $s$, which is equivalent to $C(s)>0, A(s)-$ $B(s) C^{-1}(s) B^{T}(s)>0$.

Lemma 3. Suppose that $S \in R^{n \times n}$ is positive definite and $D \in R^{n \times n}$ is symmetric. Then, for $\forall x \in R^{n}$, the following inequality holds:

$$
\lambda_{\min }\left(S^{-1} D\right) x^{T} S x \leq x^{T} D x \leq \lambda_{\max }\left(S^{-1} D\right) x^{T} S x .
$$

\section{Assumption 1}

(1) $\sum_{j=N+1}^{N+M} a_{i j}=0$ for all $i \in l_{1}, \sum_{j=1}^{N} a_{i j}=0$ for all $i \in l_{2}$

(2) The subgraph $G_{1}$ and $G_{2}$ have a directed spanning tree, respectively

Assumption 2. There exist nonnegative constants $p$ and $q$ such that nonlinear function satisfies the following equality:

$$
\left\|f_{\bar{i}}\left(x_{i}, v_{i}, t\right)-f_{\bar{i}}\left(y_{i}, z_{i}, t\right)\right\| \leq p_{\bar{i}}\left\|x_{i}-y_{i}\right\|^{2}+q_{\bar{i}}\left\|v_{i}-z_{i}\right\|^{2},
$$

where $x, y, v, z \in R^{n}, \forall t \geq 0$.

\section{Main Results}

In this section, the multiconsensus of multiagent system is analyzed. 
The distributed feedback controller of agent $i$ is designed as

$$
\begin{cases}u_{i}(t)=\alpha & \sum_{j=1}^{N} a_{i j}\left[\left(q_{j}-q_{i}\right)+\left(\dot{q}_{j}-\dot{q}_{i}\right)\right] \\ & -\alpha d_{i}\left[\left(q_{i}-q_{\bar{i}}\right)+\left(\dot{q}_{i}-\dot{q}_{\bar{i}}\right)\right], \\ & t \in[k H, k H+\delta), \\ u_{i}(t)=0, & t \in[k H+\delta,(k+1) H),\end{cases}
$$

where $H>0$ is the control period and $\delta>0$ is called the control time width.

Let $\widetilde{q}_{i}(t)=q_{i}(t)-q_{\bar{i}}(t)$ and $\widetilde{\dot{q}}_{i}(t)=\dot{q}_{i}(t)-\dot{q}_{\bar{i}}(t)$ are the measurement error of position and velocity of the $i$ th agent.

Note that if $j \in V\left(G_{k}\right)$, then $\bar{j}=k$ and $\tilde{q}_{j}(t)=q_{j}(t)-q_{\bar{k}}(t)$. We then observe

$$
\begin{aligned}
\sum_{j=1}^{N} & a_{i j}\left(q_{j}(t)-q_{i}(t)\right) \\
& =-\sum_{k=1}^{p} \sum_{j \in V\left(G_{k}\right)} l_{i j} q_{j}(t) \\
& =-\sum_{k=1}^{p} \sum_{j \in V\left(G_{k}\right)} l_{i j}\left(q_{j}(t)-q_{\bar{k}}(t)+q_{\bar{k}}(t)\right) \\
& =-\sum_{j=1}^{N} l_{i j} \tilde{x}_{j}(t)-\sum_{k=1}^{p}\left(\sum_{j \in V\left(G_{k}\right)} l_{i j}\right) q_{\bar{k}}(t) \sum_{j=1}^{N} a_{i j}\left(\tilde{x}_{j}(t)-\tilde{q}_{i}(t)\right) .
\end{aligned}
$$

Obviously, $\sum_{j=1}^{N} a_{i j}\left((\dot{q})_{j}(t)-\dot{q}_{i}(t)\right)=\sum_{j=1}^{N} a_{i j}\left(\tilde{\dot{q}}_{j}(t)-\right.$ $\left.\tilde{\dot{q}}_{i}(t)\right)$, which in turn together (1) and (2) yields the conclusion

$$
\left\{\begin{array}{l}
\widetilde{\ddot{q}}_{i}(t)=f_{\bar{i}}\left(q_{i}, \dot{q}_{i}, t\right)-f_{\bar{i}}\left(q_{\bar{i}}, \dot{q}_{\bar{i}}, t\right), \\
-\alpha \sum_{j \in V} a_{i j}\left(\widetilde{q}_{j}(t)-\widetilde{q}_{i}(t)+\widetilde{\dot{q}}_{j}(t)-\widetilde{\dot{q}}_{i}(t)\right), \\
-\alpha d_{i}\left(\widetilde{q}_{i}(t)+\widetilde{\dot{q}}_{i}(t)\right), \quad t \in[k H, k H+\delta), \\
\widetilde{\vec{q}}_{i}(t)=f_{\bar{i}}\left(q_{i}, \dot{q}_{i}, t\right)-f_{\bar{i}}\left(q_{\bar{i}}, \dot{q}_{\bar{i}}, t\right), \\
t \in[k H+\delta,(k+1) T) .
\end{array}\right.
$$

Define $\widetilde{q}(t)=\left(\widetilde{q}_{1}^{T}(t), \widetilde{q}_{2}^{T}(t), \ldots, \widetilde{q}_{N}^{T}(t)\right)^{T}$ and $\widetilde{\dot{q}}(t)=\left(\widetilde{\dot{q}}_{1}^{T}\right.$ $\left.(t), \widetilde{\dot{q}}_{2}^{T}(t), \ldots, \widetilde{\dot{q}}_{N}^{T}(t)\right)^{T}$.

Then, system (10) can be rewritten as

$$
\left\{\begin{array}{l}
\widetilde{q}(t)=F_{\bar{i}}(q(t), \dot{q}(t), t)-\widetilde{F}_{\bar{i}}\left(q_{\bar{i}}(t), \dot{q}_{\bar{i}}(t), t\right), \\
-\alpha(L+D) \otimes I_{n}(\widetilde{q}(t)+\widetilde{\dot{q}}(t)), \\
t \in[k H, k H+\delta), \\
\widetilde{\tilde{q}}_{i}(t)=F_{\bar{i}}(q(t), \dot{q}(t), t)-\widetilde{F}_{\bar{i}}\left(q_{\bar{i}}(t), \dot{q}_{\bar{i}}(t), t\right), \\
t \in[k H+\delta,(k+1) T) .
\end{array}\right.
$$

where $F(q(t), \dot{q}(t), t)=\left(f_{\bar{i}}^{H} \quad\left(q_{1}(t), \dot{q}_{1}(t), t\right), \ldots, f_{\bar{i}}^{H}\left(q_{N}\right.\right.$ $\left.\left.(t), \dot{q}_{N}(t), t\right)\right)^{H}, F(\bar{q}(t), \overline{\dot{q}}(t), t)=\left(f_{\bar{i}}^{H}\left(q_{\overline{1}}(t), \dot{q}_{\overline{1}}(t), t\right), \ldots\right.$, $\left.f_{\bar{i}}^{H}\left(q_{\bar{N}}(t), \dot{q}_{\bar{N}}(t), t\right)\right)^{H}, D=\operatorname{diag}\left\{d_{1}, \ldots, d_{N}\right\}$.

Theorem 1. Under Assumption 1 and Assumption 2, system (1) can reach the multiconsensus if the parameters meet the following conditions:

$$
\begin{gathered}
\rho I_{N}-\frac{\alpha}{2} \bar{L}-\alpha D<0, \\
\delta>\frac{\gamma}{\gamma+\eta} T,
\end{gathered}
$$

where $\rho=\max \{p, q+1\}, p=\max \left\{p_{\bar{i}}\right\}, q=\max \left\{q_{\bar{i}}\right\}, \gamma=2$ $\lambda_{\text {max }}\left(\Omega^{-1} M\right), \eta=\left(\lambda_{\text {min }}(Q) / \lambda_{\text {max }}(\Omega)\right), \Omega=\left[\begin{array}{cc}\alpha \bar{L}+2 \alpha D & I_{N} \\ I_{N} & I_{N}\end{array}\right]$, $Q=\left[\begin{array}{cc}\alpha(\bar{L} / 2)+\alpha D-p I_{N} & 0_{N} \\ 0_{N} & \alpha(\bar{L} / 2)+\alpha D-(q+1) I_{N}\end{array}\right], \quad M=$ $\left[\begin{array}{cc}p I_{N} & (\alpha / 2) \bar{L}+\alpha D \\ (\alpha / 2) \bar{L}+\alpha D & (q+1) I_{N}\end{array}\right]$, and $\bar{L}=L+L^{T}$.

Proof. It follows from (12) that

$$
\begin{gathered}
\alpha \frac{\bar{L}}{2}+\alpha D-\rho I_{N}>0, \\
{\left[\begin{array}{cc}
\alpha \bar{L}+2 \alpha D & I_{N} \\
I_{N} &
\end{array}\right]>0 .}
\end{gathered}
$$

Definite the Lyapunov function for system (12):

$$
V(t)=\frac{1}{2} \widetilde{y}^{T}(t)\left(\Omega \otimes I_{n}\right) \widetilde{y}(t)
$$

where $\Omega=\left[\begin{array}{cc}\alpha \bar{L}+2 \alpha D & I_{N} \\ I_{N} & I_{N}\end{array}\right]$ and $\tilde{y}(t)=\left(\tilde{q}^{T}(t), \tilde{\dot{q}}^{T}(t)\right)^{T}$.

In view of (8), we know that Lyapunov function (15) satisfies $V(t) \geq 0$ and $V(t)=0$ if and only if $\widetilde{q}(t)=\widetilde{\dot{q}}(t)=0$.

$$
V(t)=\frac{1}{2} \widetilde{q}^{T}\left[(\alpha \bar{L}+2 \alpha D) \otimes I_{n}\right] \widetilde{q}+\widetilde{q}^{T} \widetilde{\dot{q}}+\frac{1}{2} \widetilde{\dot{q}}^{T} \widetilde{\dot{q}}
$$

For $t \in\left[k H, t_{0}+k H+\delta\right)$, the time derivative of $V(t)$ along the trajectories of system (11) gives

$$
\begin{aligned}
\dot{V}(t)= & \widetilde{q}^{T}\left[(\alpha \bar{L}+2 \alpha D) \otimes I_{n}\right] \dot{\tilde{q}}+\dot{\tilde{q}}^{T} \widetilde{\dot{q}}+\widetilde{q}^{T} \widetilde{\ddot{q}}+\tilde{\dot{q}}^{T} \widetilde{\ddot{q}} \\
= & -\widetilde{q}^{T}\left[(\alpha L+\alpha D) \otimes I_{n}\right] \widetilde{q}+\tilde{\dot{q}}^{T}\left[\left(I_{N}-\alpha L-\alpha D\right) \otimes I_{n}\right] \tilde{\dot{q}} \\
& +(\widetilde{q}+\widetilde{\dot{q}})\left(F_{\bar{i}}(q(t), \dot{q}(t), t)-\widetilde{F}_{\bar{i}}(\bar{q}(t), \dot{\dot{q}}(t), t)\right) .
\end{aligned}
$$

Then, by (A1), we get 


$$
\begin{aligned}
\left(\widetilde{q}^{T}+\widetilde{\dot{q}}^{T}\right)\left(F_{\bar{i}}(q(t), \dot{q}(t), t)-\widetilde{F}_{\bar{i}}(\bar{q}(t), \overline{\dot{q}}(t), t)\right) & =\sum_{i=1}^{N}\left(\widetilde{q}_{i}(t)+\tilde{\dot{q}}_{i}(t)\right)^{T}\left(f_{\bar{i}}\left(q_{i}, \dot{q}_{i}, t\right)-f_{\bar{i}}\left(q_{\bar{i}}, \dot{q}_{\bar{i}}, t\right)\right) \\
& =\sum_{i=1}^{N}\left(p_{i}\left\|\widetilde{q}_{i}(t)\right\|^{2}+q_{i}\left\|\tilde{q}_{i}(t)\right\|^{2}\right) \leq \tilde{q}^{T}\left(p I_{N} \otimes I_{n}\right) \widetilde{q}+\tilde{q}^{T}\left(q I_{N} \otimes I_{n}\right) \tilde{\dot{q}} .
\end{aligned}
$$

It follows from (17) and (18) that

$$
\begin{array}{r}
\dot{V}(t) \leq-\widetilde{q}^{T}\left[\left(\alpha \frac{\bar{L}}{2}+\alpha D\right) \otimes I_{n}\right] \tilde{q}+\tilde{\dot{q}}^{T}\left(q I_{N} \otimes I_{n}\right) \tilde{\dot{q}} \tilde{\dot{q}}^{T}\left[\left(I_{N}-\alpha \frac{\bar{L}}{2}-\alpha D\right) \otimes I_{n}\right] \tilde{\dot{q}}+\tilde{q}^{T}\left(p I_{N} \otimes I_{n}\right) \tilde{q} \\
=\widetilde{q}^{T}\left[\left(-\alpha \frac{\bar{L}}{2}-\alpha D+p I_{N}\right) \otimes I_{n}\right] \tilde{q}+\widetilde{q}^{T}\left[\left(-\alpha \frac{\bar{L}}{2}-\alpha D+(q+1) I_{N}\right) \otimes I_{n}\right] \widetilde{\dot{q}}=-\widetilde{y}^{T}\left[Q \otimes I_{n}\right] \tilde{y}, \\
\left(\begin{array}{c}
\alpha(\bar{L} / 2)+\alpha D-p I_{N} \\
\quad \dot{V}(t) \leq-\frac{\lambda_{\min }(Q)}{\lambda_{\max }(\Omega)} V(t)=-\eta V(t),
\end{array}\right.
\end{array}
$$

where $\quad Q=\left(\begin{array}{cc}\alpha(\bar{L} / 2)+\alpha D-p I_{N} & 0_{N} \\ 0_{N} & \alpha(\bar{L} / 2)+\alpha D-(q+1) I_{N}\end{array}\right)$. In view of condition (12), we can get $Q>0$. It is known that Lyapunov function satisfies that

$$
\lambda_{\min }(\Omega)\|\tilde{y}(t)\|^{2} \leq V(t) \leq \lambda_{\max }(\Omega)\|\tilde{y}(t)\|^{2} .
$$

where $\eta=\left(\lambda_{\min }(Q) / \lambda_{\max }(\Omega)\right)$.

For $t \in[k H+\delta,(k+1) T)$, taking the time derivative of $V(t)$ along the trajectories of system (11)

Thus, one has

$$
\begin{aligned}
\dot{V}(t) & =\widetilde{q}^{T}\left[(\alpha \bar{L}+2 \alpha D) \otimes I_{n}\right] \dot{\tilde{q}}+\dot{\tilde{q}}^{H} \tilde{\dot{q}}+\widetilde{q} \widetilde{\dot{q}}+\widetilde{\ddot{q}}^{T} \tilde{\ddot{q}} \leq \widetilde{y}^{T}\left(\left(\begin{array}{cc}
p I_{N} & \frac{\alpha}{2} \bar{L}+\alpha D \\
\frac{\alpha}{2} \bar{L}+\alpha D & (q+1) I_{N}
\end{array}\right) \otimes I_{n}\right) \\
\tilde{y} & \leq \widetilde{y}^{T}\left[M \otimes I_{n}\right] \tilde{y} \leq 2 \lambda_{\max }\left(\Omega^{-1} M\right) V(t)=\gamma V(t),
\end{aligned}
$$

where $M=$
$\lambda_{\max }\left(\Omega^{-1} M\right)$.$\left[\begin{array}{cc}p I_{N} & (\alpha / 2) \bar{L}+\alpha D \\ (\alpha / 2) \bar{L}+\alpha D & (q+1) I_{N}\end{array}\right]$ and $\quad \gamma=2$

Then, by the above differential inequality (21) and (22), we have the following results:

(1) For $0<t<\delta, V(t) \leq V(0) e^{-\eta t}$ and $V(\delta) \leq V(0) e^{-\eta t}$

(2) For $\delta \leq t<H, V(t) \leq V(\delta) e^{(\gamma(t-\delta))} \leq V(0) e^{(\gamma(t-\delta)-\eta \delta)}$ and $V(H) \leq V(0) e^{(\gamma(H-\delta)-\eta \delta)}$

(3) $H \leq t<H+\delta, \quad V(t) \leq V(H) e^{(-\eta(t-H))} \leq V(0)$ $e^{(\gamma(H-\delta)-\eta \delta-\eta(t-H))}, \quad$ and $\quad V(H+\delta) \leq V(0)$ $e^{(\gamma(H-\delta)-2 \eta \delta)}$

(4) $H+\delta \leq t<2 H, V(t) \leq V(H+\delta) e^{(\gamma(t-T H-\delta))} \leq V(0)$ $e^{(\gamma(H-\delta)+\gamma(t-H-\delta)-2 \eta \delta)} \quad$ and $V(2 H) \leq V(0)$ $e^{(\gamma(H-\delta)+\gamma(H-\delta)-2 \eta \delta)}=V(0) e^{(2 \gamma(H-\delta)-2 \eta \delta)}$

Thus, one has For $k H \leq t \leq k H+\delta$,
$V(t) \leq V(k H) \exp (-\eta(t-k H))$

$$
\begin{aligned}
& \leq V(0) \exp (k \gamma(H-\delta)-k \eta \delta-\eta(t-k H)) \\
& \leq V(0) \exp \left(-\frac{\eta \delta-\gamma(H-\delta)}{H}(t-\delta)\right) \exp \left(\frac{H-\delta}{H} \gamma \delta\right) \\
& \leq V(0) \exp \left(-\frac{\eta \delta-\gamma(H-\delta)}{H}(t-\delta)\right) \exp (\gamma(H-\delta)) .
\end{aligned}
$$

For $k H+\delta \leq t<(k+1) H$,

$$
\begin{aligned}
V(t) & \leq V(k H+\delta) e^{\gamma(t-k H-\delta)} \\
& \leq V(0) \exp (k+1) \gamma((H-\delta-k+1) \eta \delta) \\
& \leq V(0) \exp \left(-\frac{\eta \delta-\gamma(H-\delta)}{H}(t-\delta)\right) \exp (\gamma(H-\delta)) .
\end{aligned}
$$


If $\delta>(\gamma / \gamma+\eta) H$, then defining $\xi=(\eta \delta-(H-$ $\delta) \gamma / H)>0$. Let $K=V(0) \exp ((\eta \delta-\gamma(H-\delta) / H) \delta) \exp$ $(\gamma(H-\delta))$, and from the above analysis, we can draw the following conclusions:

$$
V(t) \leq K e^{-(\eta \delta-\gamma(H-\delta) / H) t} .
$$

Therefore, the multiconsensus can be achieved. The proof is completed.

Remark 1. Under the condition of Theorem 1, the multiconsensus of system (1) can be achieved globally exponentially with the presented law.

Remark 2. When each agent has the identical nonlinear function, all the agents have the same virtual leader. The dynamics of the virtual leader can be described as

$$
\ddot{q}_{r}(t)=f\left(q_{r}, \dot{q}_{r}, t\right) .
$$

Under the intermittent control, system (1) can reach consensus.

Theorem 2. Under Assumption 1 and Assumption 2, system (1) can reach the multiconsensus if the parameter meets the following conditions:

$$
\begin{aligned}
& \text { (1) } I_{N}-\frac{\alpha}{2} \bar{L}-\alpha D<0, \\
& \text { (2) } \delta>\frac{\gamma}{\gamma+\eta} T
\end{aligned}
$$

where $\rho=\max \left\{\rho_{1}, \rho_{2}+1\right\}, \quad \gamma=2 \lambda_{\max }\left(\Omega^{-1} M\right), \quad \eta=\left(\lambda_{\min }\right.$ $\left.(Q) / \lambda_{\max }(\Omega)\right), \quad \bar{L}=L+L^{T}, \quad \Omega=\left[\begin{array}{cc}\alpha \bar{L}+2 \alpha D & I_{N} \\ I_{N} & I_{N}\end{array}\right], \quad Q=$ $\left[\begin{array}{cc}\alpha(\bar{L} / 2)+\alpha D & 0_{N} \\ 0_{N} & \alpha(\bar{L} / 2)+\alpha D-I_{N}\end{array}\right], \quad$ and $\quad M=$ $\left[\begin{array}{cc}0 & (\alpha / 2) \bar{L}+\alpha D \\ (\alpha / 2) \bar{L}+\alpha D & I_{N}\end{array}\right]$.

When $f\left(q_{i}, \dot{q}_{i}, t\right)=0$, the dynamics of the $i$ th agent can be described as follows:

$$
\ddot{q}_{i}(t)=u_{i}(t) .
$$

Then, (28) shows that the velocity of virtual leader is constant. Under protocol (8), let $\tilde{q}_{i}(t)=q_{i}(t)-q_{\bar{i}}(t)$, $\widetilde{\dot{q}}_{i}(t)=\dot{q}_{i}(t)-\dot{q}_{\bar{i}}(t)$, then the closed-loop system (28) becomes:

$$
\begin{cases}\tilde{\ddot{q}}_{i}(t)=-\alpha \sum_{j \in V} a_{i j}\left(\tilde{q}_{j}(t)-\tilde{q}_{i}(t)+\tilde{\dot{q}}_{j}(t)-\tilde{\dot{q}}_{i}(t)\right)-\alpha d_{i}\left(\widetilde{q}_{i}(t)+\tilde{\dot{q}}_{i}(t)\right), & t \in[k H, k H+\delta), \\ \tilde{\dot{q}}_{i}(t)=0, & t \in[k H+\delta,(k+1) H) .\end{cases}
$$

where $i=1,2, \ldots, N$. System (29) can be rewritten as

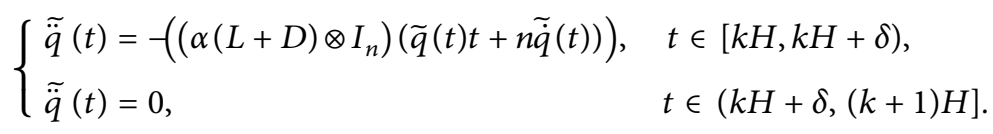

The proof of this part is similar to that of Theorem 1, which is omitted here.

\section{Numerical Simulations}

In this part, the effectiveness of presented theoretical results has been proven by numerical examples. The multiconsensus of second-order agent systems has been studied. Actually, seven agents can form a second-order system which is split into two clusters $v_{1}=\{1,2,3\}$ and $v_{2}=\{4,5,6,7\}$. If $i \in \nu_{1}$, then $\widehat{i}=1$; and if $i \in \nu_{2}$, then $\widehat{i}=2$.

The Laplacian matrix is given as follows:

$$
L=\left[\begin{array}{ccccccc}
3 & -3 & 0 & 1 & 0 & 0 & -1 \\
-2 & 3 & -1 & -1 & 0 & 0 & 1 \\
-1 & 0 & 1 & 0 & 0 & 0 & 0 \\
1 & -1 & 0 & 2 & 0 & 0 & -2 \\
0 & 0 & 0 & -1 & 1 & 0 & 0 \\
0 & 0 & 0 & 0 & -1 & 1 & 0 \\
-1 & 1 & 0 & -2 & 0 & -1 & 3
\end{array}\right],
$$




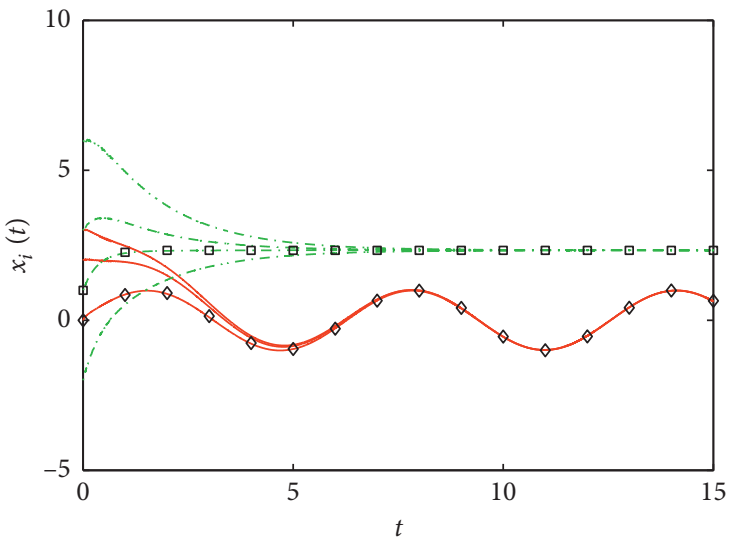

(a)

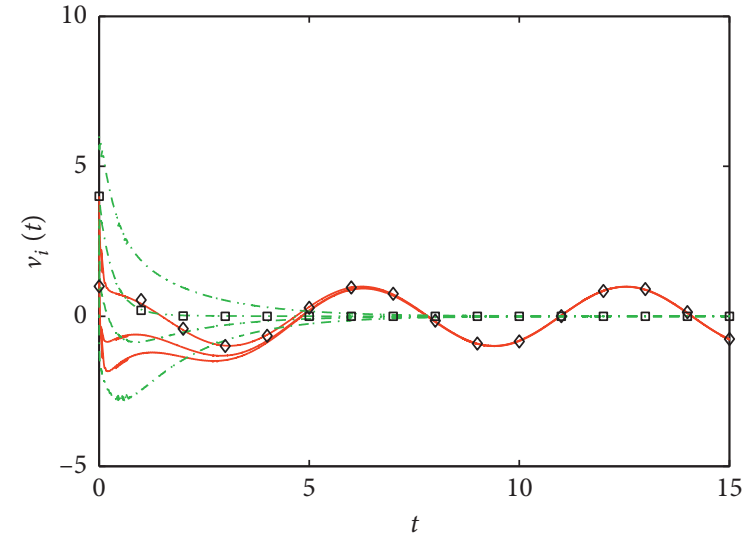

(b)

Figure 1: The trajectories of the agents.

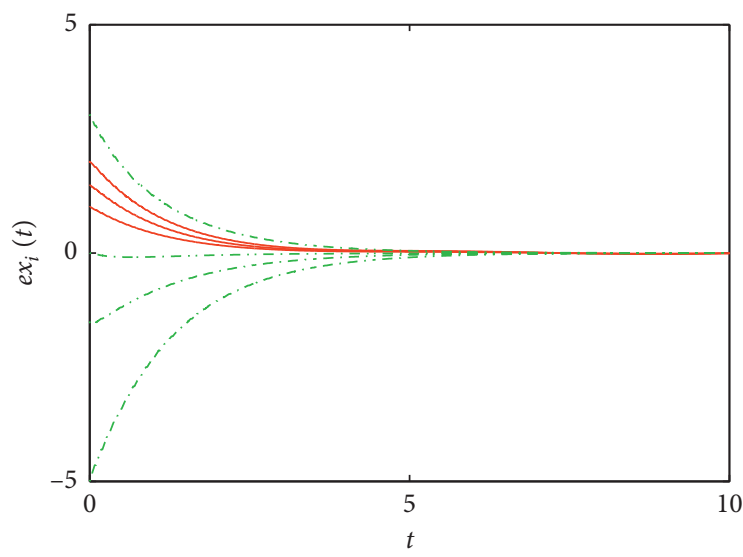

(a)

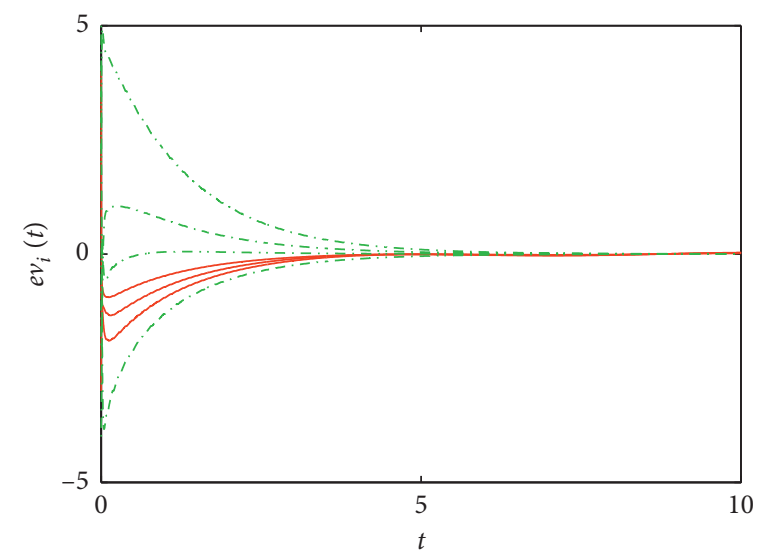

(b)

Figure 2: The error trajectories of the agents.

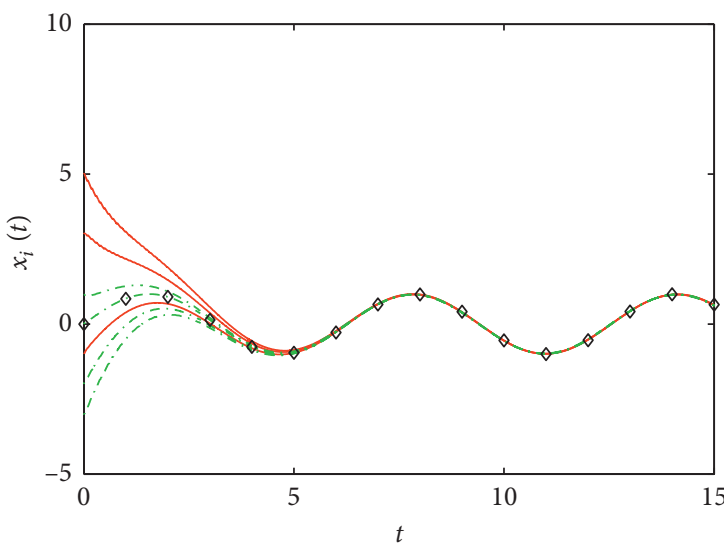

(a)

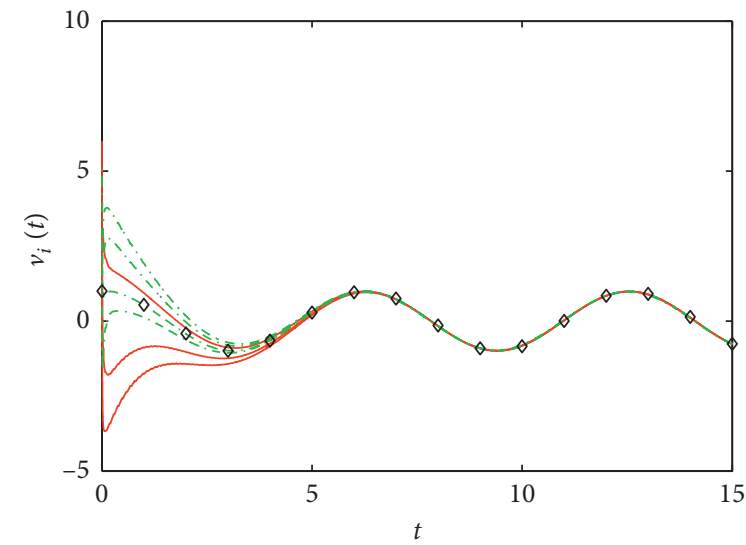

(b)

Figure 3: The trajectories of the agents. 


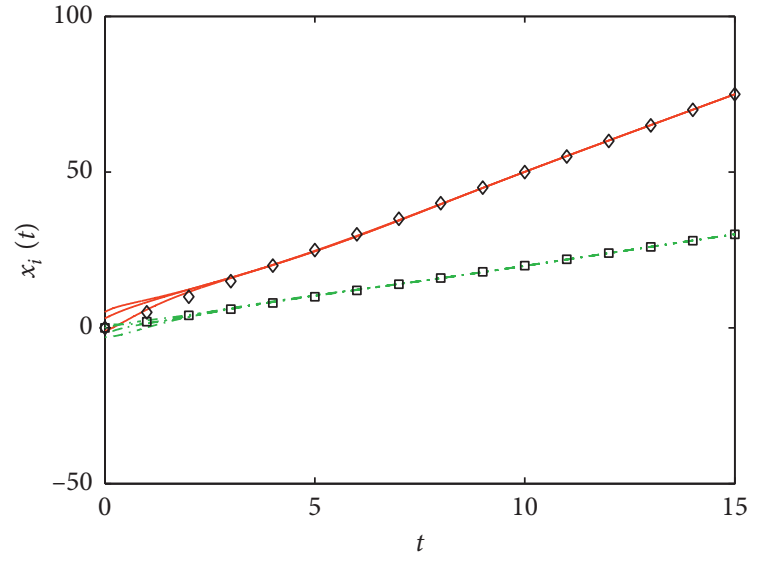

(a)

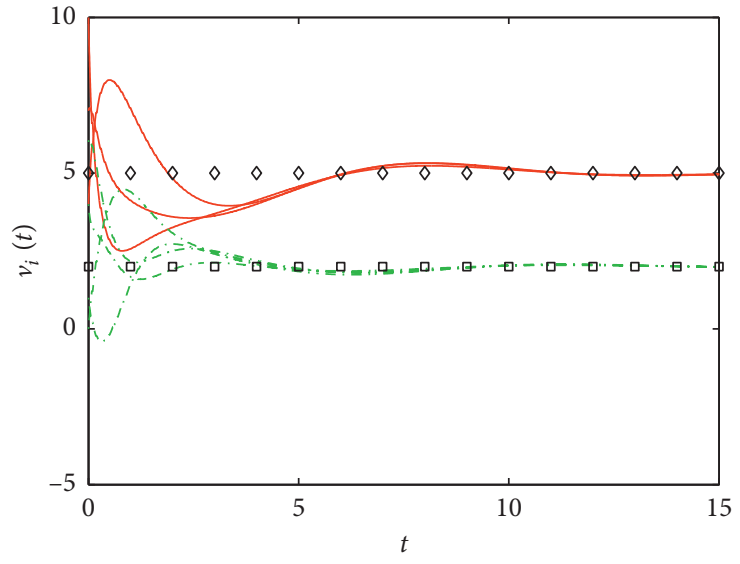

(b)

Figure 4: The trajectories of the agents.

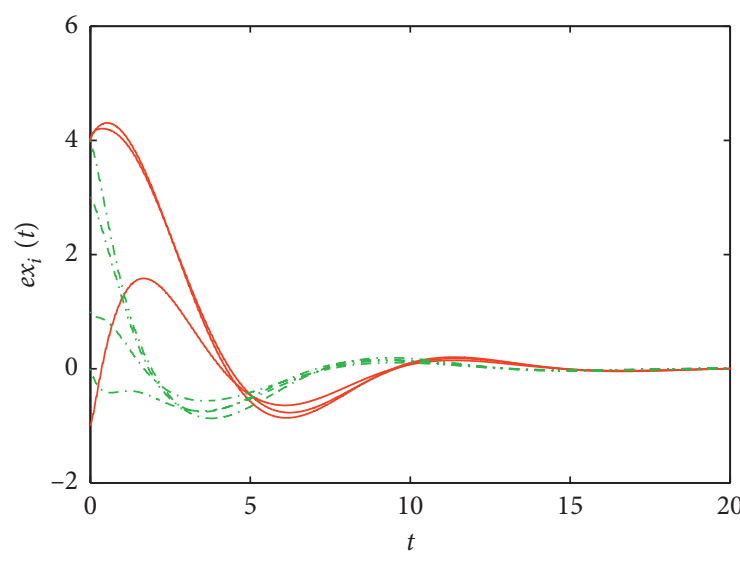

(a)

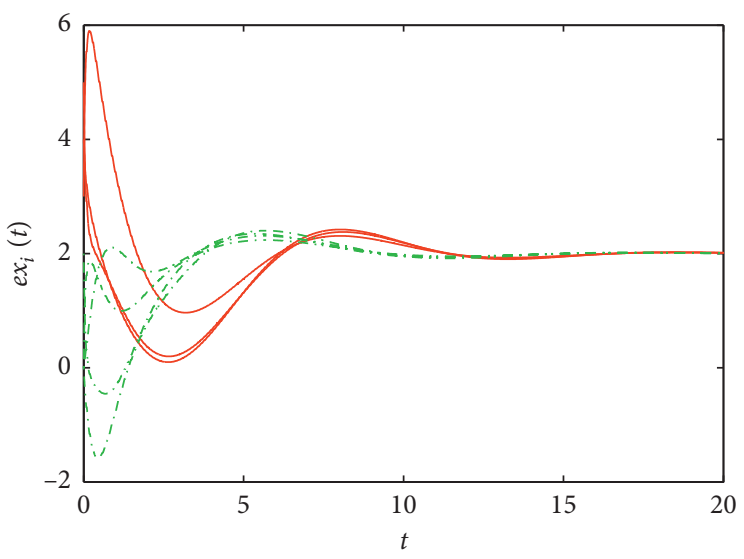

(b)

Figure 5: The error trajectories of the agents.

Case 1. Set $f_{1}\left(x_{i}, v_{i}, t\right)=-x_{i}$ and $f_{2}\left(x_{i}, v_{i}, t\right)=-3 v_{i}$. By Assumption 2, $p_{1}=1, q_{1}=0$, and $p_{2}=0, q_{2}=3$. Then, $\rho=4$. In view of condition (12), we can choose $\alpha=15$. By condition (13), we choose $\delta=0.08$. The initial position and velocity of the virtual leader are given as follows:

$$
\begin{aligned}
& \left(q_{1}(0), \dot{q}_{1}(0)\right)=(0,1), \\
& \left(q_{2}(0), \dot{q}_{2}(0)\right)=(1,4) .
\end{aligned}
$$

Figure 1 shows the states and velocity of the virtual leaders and followers. It is easy to see that the multiconsensus of systems (1) can be received. The error tracks of the agents are depicted in Figure 2.

When $f_{\overline{1}}\left(x_{i}, v_{i}, t\right)=f_{\overline{2}}\left(x_{i}, v_{i}, t\right)=-x_{i}$, as shown in Figure 3 , the consensus problem of system (1) is indeed solved.

Case 2. Let $f_{\overline{1}}\left(x_{i}, v_{i}, t\right)=0$ and $f_{\overline{2}}\left(x_{i}, v_{i}, t\right)=0$. The initial velocity of the virtual leader are given as follows: $v_{\overline{1}}(0)=2$ and $v_{\overline{2}}(0)=5$. The multiconsensus of the systems can be achieved. As a special case, when the initial velocity of the virtual leader is same, the system reaches consensus. The simulation result is shown in Figures 4 and 5, which show that the multiconsensus can be achieved.

\section{Conclusion}

In this paper, we have studied on multiconsensus of secondorder multiagent systems with nonlinear dynamics. For the realization of multiconsensus, a distributed protocol based on intermittent relative information has been proposed. Some of sufficient conditions have been given to ensure that the states of all agents could reach more consistent values. In this paper, two simulation examples are given and applied to the effectiveness of theoretical results.

\section{Data Availability}

All data included in this study are available upon request by contact with the corresponding author. 


\section{Conflicts of Interest}

The authors declare no potential conflicts of interest with respect to the research, authorship, and/or publication of this article.

\section{Acknowledgments}

This work was supported in part by the Natural Science Foundation of Hubei Province (2016CFB514) and the Research Fund for the Doctoral Program of Hubei University of Technology (nos. BSQD2015044, BSQD2015024, BSQD2019050).

\section{References}

[1] X.-W. Jiang, X.-Y. Chen, T.-W. Huang, and H.-C. Yan, "Output tracking control of single-input multi-output systems over an erasure channel," IEEE Transactions on Cybernetics, pp. 1-9, 2020.

[2] H. Su, H. Wu, and J. Lam, "Positive edge-consensus for nodal networks via output feedback," IEEE Transactions on Automatic Control, vol. 64, no. 3, pp. 1244-1249, 2019.

[3] J.-W. Sun, G.-Y. Han, Z. Zeng, and Y.-F. Wang, "Memristorbased neural network circuit of full-function pavlov associative memory with time delay and variable learning rate," IEEE Transactions on Cybernetics, pp. 1-11, 2019.

[4] J. Sun, Y. Wu, G. Cui, and Y. Wang, "Finite-time real combination synchronization of three complex-variable chaotic systems with unknown parameters via sliding mode control," Nonlinear Dynamics, vol. 88, no. 3, pp. 1677-1690, 2017.

[5] G.-H. Xu, F. Qi, Q. Lai, and H. Ho-Ching Iu, "Fixed time synchronization control for bilateral teleoperation mobile manipulator with nonholonomic constraint and time delay," IEEE Transactions on Circuits and Systems II: Express Briefs, p. 1, 2020.

[6] J. Yu and L. Wang, "Group consensus in multi-agent systems with switching topologies and communication delays," Systems \& Control Letters, vol. 59, no. 6, pp. 340-348, 2010.

[7] J. Yu and L. Wang, "Group consensus of multi-agent systems with directed information exchange," International Journal of Systems Science, vol. 43, no. 2, pp. 334-348, 2012.

[8] S. Monaco and L. Ricciardi Celsi, "On multi-consensus and almost equitable graph partitions," Automatica, vol. 103, pp. 53-61, 2019.

[9] Y. Feng, S. Xu, and B. Zhang, "Group consensus control for double-integrator dynamic multiagent systems with fixed communication topology," International Journal of Robust and Nonlinear Control, vol. 24, no. 3, pp. 532-547, 2014.

[10] X.-Q. Lu, A. Francis, and S.-H. Chen, "Cluster consensus of nonlinearly coupled multi-agent systems in directed graphs," Chinese Physics Letters, vol. 27, no. 5, pp. 1089-1095, 2010.

[11] X.-Q. Lu, F. Austin, and S.-H. Chen, "Cluster consensus of second-order multi-agent systems via pinning control," Chinese Physics B, vol. 19, no. 12, pp. 1-7, Article ID 120506, 2010.

[12] X. Liao and L. Ji, "On pinning group consensus for dynamical multi-agent networks with general connected topology," Neurocomputing, vol. 135, pp. 262-267, 2014.

[13] H. Zhao and J. H. Park, "Group consensus of discrete-time multi-agent systems with fixed and stochastic switching topologies," Nonlinear Dynamics, vol. 77, no. 4, pp. 12971307, 2014.

[14] J. Qin and C. Yu, "Cluster consensus control of generic linear multi-agent systems under directed topology with acyclic partition," Automatica, vol. 49, no. 9, pp. 2898-2905, 2013.

[15] G. Wang and Y. Shen, "Second-order cluster consensus of multi-agent dynamical systems with impulsive effects," Communications in Nonlinear Science and Numerical Simulation, vol. 19, no. 9, pp. 3220-3228, 2014.

[16] G. Wen, Z. Li, Z. Duan, and G. Chen, "Distributed consensus control for linear multi-agent systems with discontinuous observations," International Journal of Control, vol. 86, no. 1, pp. 95-106, 2013.

[17] L. Xiao and X. Liao, "Periodic intermittent consensus of second-order agents networks with nonlinear dynamics," International Journal of Control, Automation and Systems, vol. 12, no. 1, pp. 23-28, 2014.

[18] Y. Gao and L. Wang, "Consensus of multiple double-integrator agents with intermittent measurement," International Journal of Robust and Nonlinear Control, vol. 20, pp. 11401155, 2010.

[19] G. Wen, Z. Duan, W. Yu, and G. Chen, "Consensus of secondorder multi-agent systems with delayed nonlinear dynamics and intermittent communications," International Journal of Control, vol. 86, no. 2, pp. 322-331, 2013.

[20] A. Hu, J. Cao, and M. Hu, "Consensus of leader-following multi-agent systems in time-varying networks via intermittent control," International Journal of Control, Automation and Systems, vol. 12, no. 5, pp. 969-976, 2014.

[21] G.-H. Xu, M. Xu, M.-F. Ge, T.-F. Ding, F. Qi, and M. Li, "Distributed event-based control of hierarchical leader-follower networks with time-varying layer-to-layer delays," Energies, vol. 13, no. 7, p. 1808, 2020.

[22] Z. Peng, D. Wang, G. Sun, and H. Wang, "Distributed cooperative stabilisation of continuous-time uncertain nonlinear multi-agent systems," International Journal of Systems Science, vol. 45, no. 10, pp. 2031-2041, 2014.

[23] H. Su, G. Jia, and M. Z. Q. Chen, "Semi-global containment control of multi-agent systems with intermittent input saturation," Journal of The Franklin Institute, vol. 352, no. 9, pp. 3504-3525, 2015.

[24] G. Wen, Z. Peng, A. Rahmani, and Y. Yu, "Distributed leaderfollowing consensus for second-order multi-agent systems with nonlinear inherent dynamics," International Journal of Systems Science, vol. 45, no. 9, pp. 1892-1901, 2014. 